\title{
$17 \beta$-estradiol reduced depressive and somatic symptoms in perimenopausal women
}

Soares CN, Almeida OP,Joffe H, et al. Efficacy of estradiol for the treatment of depressive disorders in perimenopausal women. A double-blind, randomized, placebo-controlled trial. Arch Gen Psychiatry 2001 Jun;58:529-34.

\section{QUESTION: In perimenopausal women with clinically important depressive disorders, does $17 \beta$-estradiol decrease depressive symptoms?}

\section{Design}

12 week randomised (allocation concealed*), blinded \{clinicians, patients, outcome assessors, and statisticians $\} \nmid, *$ placebo controlled trial.

\section{Setting}

A gynaecological clinic and psychiatric outpatient clinic in São Paulo, Brazil.

\section{Patients}

50 women who were 40 to 55 years of age (mean age $50 \mathrm{y}$ ); had a history of menstrual cycle irregularity or amenorrhoea for $<12$ months; had a serum concentration of follicle-stimulating hormone $>25 \mathrm{IU} / \mathrm{l}$; and had been diagnosed with major depressive disorder, dysthymic disorder, or minor depressive disorder. Exclusion criteria were medical illness; hormone replacement therapy or psychoactive drug use in the previous 3 months; or presence of psychotic features or suicidal or severe aggressive behaviour. Follow up was $90 \%$.

\section{Intervention}

Patients were allocated to a $17 \beta$-estradiol patch, $100 \mu \mathrm{g}$ (Systen/Evorel, Janssen-Cilag Laboratories, São Paulo, Brazil) $(n=25)$, or a placebo patch $(n=25)$.

\section{Main outcome measures}

Severity of depressive symptoms measured by the Montgomery-Asberg Depression Rating Scale (MADRS) and severity of perimenopausal symptoms measured by the Blatt-Kupperman Menopausal Index (BKMI). Remission of depression was achieved if the MADRS score was $<10$. A decrease of $\geqslant 50 \%$ from the baseline BKMI score was considered a significant improvement in somatic symptoms.

\section{Main results}

Analysis was by intention to treat. At 12 weeks, MADRS scores decreased more from baseline in women who received $17 \beta$-estradiol than in those who received placebo $(-16.36 v-4.16,\{95 \%$ CI for the 12.2 difference in change from baseline 8.4 to 16.0$\} \$, p<0.001)$. More women who received estradiol had remission of depression $(\mathrm{p}=0.001)$ and $\mathrm{a} \geqslant 50 \%$ decrease in BKMI scores $(\mathrm{p}=0.005)$ than did women who received placebo (table). The groups did not differ for adverse events. At the end of a 4 week washout period, MADRS scores remained lower than those at baseline in the estradiol group $(\mathrm{p}<$ $0.001)$ and were as severe as those at baseline in the placebo group $(\mathrm{p}=0.07)$.

\section{Conclusion}

In perimenopausal women with clinically important depressive disorders, $17 \beta$-estradiol decreased depressive and somatic symptoms.

*See glossary.

$\ddagger$ CI calculated from data in article. $\dagger$ Information provided by author.

Source of funding: Fundação de Amparo à Pesquisa do Estado de São Paulo

(FAPESP)-São Paulo

Research Foundation.

For correspondence: $D$ CN Soares, Perinatal and Reproductive Psychiatry Clinical Research Program, Massachusetts General Hospital, Harvard Medical School, 15 Parkman Street, WACC 812, Boston, $M A$ 02114, USA.Fax +1 6177243028 .

17ß-estradiol v placebo patch for depressive disorders in perimenopausal women§

\begin{tabular}{|c|c|c|c|c|}
\hline Outcomes at 12 weeks & $17 \beta$-estradiol & Placebo & RBI $(95 \% \mathrm{Cl})$ & NNT (Cl) \\
\hline Remission of depression & $68 \%$ & $20 \%$ & $71 \%$ (38 to 87 ) & 3 (1 to 5$)$ \\
\hline $\begin{array}{l}\geqslant 50 \% \text { decrease from } \\
\text { baseline in BKMI score }\end{array}$ & $68 \%$ & $28 \%$ & $59 \%$ (23 to 80 ) & 3 (2 to 8 ) \\
\hline
\end{tabular}

§BKMI = Blatt-Kupperman Menopausal Index. Other abbreviations defined in glossary; RBI, NNT, and $\mathrm{Cl}$ calculated from data in article.

\section{COMMENTARY}

In the landmark study from Brazil by Soares $e t a l$, of the 50 perimenopausal women enrolled, 52\% met the Diagnostic and Statistical Manual of Mental Disorders, 4th edition (DSM-IV), criteria for major depressive disorder, 26\% for minor depressive disorder, and $22 \%$ for dysthymic disorder. The study had a 12 week treatment phase and a 4 week washout phase. A relatively high dose of oestrogen $(100 \mu \mathrm{g})$ alone was used. Progesterone (which is the standard of care in women with a uterus or endometrium) was not used. $68 \%$ of women treated with transdermal $17 \beta$-estradiol had remission of depression, regardless of DSM-IV diagnosis, compared with $20 \%$ of women in the placebo group.

The Massachusetts Women's Health Study, a prospective 5 year observational trial, found no link between the onset of natural menopause and an increased risk for depression. ${ }^{1}$ However, women with a lengthy perimenopause apparently had moderately increased rates of depressive symptoms. Therefore, it is not surprising that mood disturbances, which may be higher in symptomatic perimenopausal women, would respond to oestrogen.

We need to determine which women with depressive symptoms benefit from oestrogen alone, oestrogen with a conventional antidepressant, or antidepressant treatment alone. Transdermal estradiol may be the best initial first-line treatment in women who have perimenopausal symptoms and minor mild-to-moderate mood symptoms, particularly if they do not have a uterus and do not need a progestin. Standard antidepressant treatment remains the first line of treatment for perimenopausal women with major depressive disorders alone.

We are moving beyond viewing oestrogen as only a reproductive hormone to viewing it as a neural hormonal agent with effects on mood and cognition.

Holly L Thacker, MD Cleveland Clinic Foundation Cleveland, Ohio, USA

1 Avis NE, Brambilla D, McKinlay SM, et al. A longitudinal analysis of the association between menopause and depression. Results from the Massachusetts Women's Health Study. Ann Epidemiol 1994;4:214-20. 